\title{
WHOLE EARTH TELESCOPE OBSERVATIONS OF THE DAV WHITE DWARF G226-29
}

\author{
S. O. Kepler, ${ }^{1,2}$ O. Giovannini, ${ }^{1}$ M. A. Wood, ${ }^{3}$ R. E. Nather,${ }^{4}$ D. E. Winget, ${ }^{4}$ A. Kanaan, ${ }^{4}$ \\ S. J. Kleinman, ${ }^{4}$ P. A. Bradley, ${ }^{4,5}$ J. L. Provencal, ${ }^{4,6}$ J. C. Clemens, ${ }^{4,7}$ C. F. Claver, ${ }^{4,8}$ \\ T. K. Watson, ${ }^{4}$ K. Yanagida, ${ }^{4}$ K. Krisciunas, ${ }^{9}$ T. M. K. Marar, ${ }^{10}$ S. Seetha, ${ }^{10}$ \\ B. N. AshoKa, ${ }^{10}$ E. Leibowitz, ${ }^{11}$ H. Mendelson,,${ }^{11}$ T. Mazeh, ${ }^{11}$ P. MosKaliK, ${ }^{12}$ \\ J. Krzesiński, ${ }^{13}$ G. Pajdosz, ${ }^{13}$ S. Zola, ${ }^{14}$ J.-E. Solheim, ${ }^{15}$ P.-I. Emanuelsen, ${ }^{16}$ \\ N. Dolez, ${ }^{16,17}$ G. Vauclair, ${ }^{16}$ M. Chevreton, ${ }^{18}$ J.-R. Fremy, ${ }^{16}$ \\ M. A. Barstow, ${ }^{19}$ A. E. SAnsom, ${ }^{19}$ R. W. TWEedy, ${ }^{19}$ \\ D. T. Wickramasinghe, ${ }^{20}$ L. Ferrario, ${ }^{20}$ \\ D. J. Sullivan, ${ }^{21}$ A. J. VAN Der PeEt, ${ }^{21}$ \\ D. A. H. BuCKLey, ${ }^{22}$ AND A.-L. ChEN ${ }^{22}$ \\ Received 1994 October 25; accepted 1994 December 13
}

\begin{abstract}
We observed G226-29 for $121 \mathrm{hr}$ in 1992 February and confirm the presence of the three previously identified frequencies close to $109 \mathrm{~s}$. We find no evidence of other pulsation periods down to our noise level of about 0.35 millimodulation amplitudes. The presence of only one triplet pulsation mode in G226-29 and its effective temperature near the blue edge of the instability strip identify the observed triplet of modes near 109 $\mathrm{s}$ as rotationally split components of the $k=1, l=1$ mode. With the mode identification, we derived a rotation period of $8.9 \mathrm{hr}$ and an inclination of the pulsation axis of $70^{\circ}-75^{\circ}$ to our line of sight.
\end{abstract}

Subject headings: stars: individual (G226-29) - stars: oscillations - stars: rotation - white dwarfs

\section{INTRODUCTION}

G226-29 (DN Dra, LP 101-148, WD 1647+591) is the brightest known pulsating DA white dwarf (DAV or ZZ Ceti star), with $m_{v}=12.22$. In addition, G226-29 has a large parallax of 0".0827 \pm 0 ".0046 (Harrington \& Dahn 1980); at just over 12 pc, it is the closest ZZ Ceti star. McGraw \& Fontaine (1980) discovered the variability of G226-29 in 1980 April using the MMT telescope. Kepler, Robinson, \& Nather (1983, hereafter KRN) used $65 \mathrm{hr}$ of temporal spectroscopy (time-series photometry measurements) to untangle the light curve and interpret the variations as an equally spaced triplet with periods near $109 \mathrm{~s}$. The outer peaks have amplitudes near $3 \mathrm{mma}^{23}$ and are separated by $\delta f=16.14 \mu \mathrm{Hz}$ from the central peak, which has an amplitude of $1.7 \mathrm{mma}$. G226-29 has the simplest mode structure, the second smallest overall pulsation amplitude, and the shortest dominant period of any pulsating white dwarf, making it a unique object.

\footnotetext{
${ }^{1}$ Instituto de Fisica, Universidade Federal do Rio Grande do Sul, 91501970 Porto Alegre, RS, Brazil.

${ }^{2}$ kepler@if1.if.ufrgs.br.

${ }^{3}$ Department of Physics and Space Sciences, Florida Institute of Technology, 150 W. University Boulevard, Melbourne, FL 32901

4 McDonald Observatory and Department of Astronomy, the University of Texas at Austin, Austin, TX 78712

5 Current address: X-2 M.S. B220, Los Alamos National Laboratory, Los Alamos, NM 87545.

${ }^{6}$ Current address: Department of Physics and Astronomy, University of Delaware, Newark, DE 19716.

${ }^{7}$ Hubble Fellow; current address: Department of Physics and Astronomy, Iowa State University, Ames, IA 50211.

${ }^{8}$ Guest Observer, Mauna Kea, HI.

9 Joint Astronomy Centre, 660 N.A'Ohokı Place, Hilo, HI 96720.

10 Technical Physics Division, ISRO Satellite Centre, Bangalore 560017 , India.

${ }^{11}$ Wise Observatory, the Sackler Faculty of Exact Sciences, Tel Aviv University, Tel Aviv 69978, Israel.

12 Copernicus Astronomical Center, Polish Academy of Sciences, 00-716 Warsaw, Poland.
}

Several recent spectroscopic studies also show G226-29 to be one of the hottest of the $\mathrm{ZZ}$ Ceti stars, suggesting that we may be observing it as it enters the instability strip. The effective temperature of this star is not settled, because one can derive two different effective temperatures for a given gravity using optical spectra (see Daou et al. 1990 for a discussion) and also because of uncertainties over the best convective efficiency to use in model atmospheres (Bergeron, Wesemael, \& Fontaine 1992). Fontaine et al. (1992) derive $T_{\text {eff }}=13,630 \pm 200 \mathrm{~K}$ and $\log g=8.18 \pm 0.05$, corresponding to a stellar mass of $0.70 \pm 0.03 M_{\odot}$, based on high signal-to-noise optical spectra and ML 2 model atmospheres. This effective temperature places G226-29 near the blue edge of their ZZ Ceti instability strip. Kepler \& Nelan (1993) use published IUE spectra and optical photometry to derive $T_{\text {eff }}=12,120 \pm 11 \mathrm{~K}$ (assuming $\log g=8.0$ ); their ZZ Ceti instability strip is much cooler, $12,640 \mathrm{~K} \geq T_{\text {eff }} \geq 11,740 \mathrm{~K}$. Finally, Koester \& Allard (1993)

\footnotetext{
13 Institute of Physics, Cracow Pedagogical University, 30-084 Krakow, Poland

${ }^{14}$ Astronomical Observatory, Jagiellonian University, 30-224 Krakow, Poland.

${ }^{15}$ Institutt for Matematiske Realfag, Universitetet i Tromso 9037 Tromso, Norway.

${ }^{16}$ Observatoire Midi-Pyrenees, 14 Avenue E. Belin, 31400 Toulouse, France.

17 C.E.R.F.A.C.S, 31400 Toulouse, France

18 Observatoire de Paris-Meudon, F-92195, Principal Cedex, France.

19 Department of Physics and Astronomy, University of Leicester, LE1 7RH, UK

${ }^{20}$ Department of Mathematics, Australian National University, Canberra Australia.

${ }^{21}$ Department of Physics, Victoria University of Wellington, Wellington, New Zealand.

22 Department of Astronomy, University of Cape Town, Rondebosch 7700, Cape Province, South Africa.

${ }_{23}$ The unit mma represents millimodulation amplitude, which corresponds to $1 / 1.086 \mathrm{mmag}$, formerly known as the millifractional amplitude.
} 
use the $\operatorname{Ly} \alpha$ line to derive a parallax consistent solution of $T_{\text {eff }}=12,040 \mathrm{~K}$ and $\log g=8.12$. The general agreement of $\log$ $g$ suggests the mass is around $0.70 \mathrm{M}_{\odot}$. In relation to the other ZZ Cetis, we have two choices for the effective temperature; it is close to either $13,600 \mathrm{~K}$ or $12,100 \mathrm{~K}$. In absolute terms, the effective temperature is $12,900 \pm 750 \mathrm{~K}$.

The very short (109 s) period triplet poses problems for seismological interpretations of the structure of G226-29, especially given the belief during the 1980 s that ZZ Ceti stars have hydrogen layer masses of $10^{-8} M_{*}$ or smaller (Winget et al. 1982; Bradley, Winget, \& Wood 1989). As KRN point out, we could be seeing an $l=3, k=1$ mode on a $\sim 0.6 M_{\odot}$ white dwarf or an $l=1, k=1$ mode on a $\sim 1 M_{\odot}$ white dwarf, depending on whether the stellar mass is near the average or if we see all three members of a rotationally split $l=1$ mode. They did not consider the possibility of G226-29 having a hydrogen layer mass greater than $10^{-7}$ to $10^{-8} M_{*}$, given the then-prevailing theoretical evidence that white dwarfs with "thick" hydrogen layers are pulsationally stable. The recent $\log g$ determinations rule out a massive $1 M_{\odot}$ white dwarf, and recent theory shows there is no sensitivity of the blue edge to the hydrogen layer mass (Cox et al. 1987; Bradley \& Winget 1994; Fontaine, Brassard, \& Wesemael 1994).

Given the unique nature of G226-29, we observed it with the Whole Earth Telescope (WET; Nather et al. 1990) during the 1992 spring observing run. We searched for extra components of the $109 \mathrm{~s}$ triplet and also sought to reduce the noise level so we could look for other periodicities, which would provide additional constraints on the structure of G226-29.

\section{OBSERVATIONS}

G226-29 $\left[\alpha=16^{\mathrm{h}} 48^{\mathrm{m}} 25^{\mathrm{s}}, \delta=+59^{\circ} 03^{\prime} 24^{\prime \prime}(2000)\right]$ was a secondary target for northern hemisphere observers during the 1992 February WET observing run. We collected $121 \mathrm{hr}$ of data in 14 days from four observatories well distributed in longitude (see Table 1 for our observing journal). Because G226-29 was a second priority object, our duty cycle was only $39 \%$. Our distribution of observing resources during the run was such that observers in Poland garnered over $60 \%$ of the data, including five runs of $9 \mathrm{hr}$ or more.

Our data were taken with two-star photometers (Nather 1973) under photometric conditions verified by simultaneous observations of a comparison star. The observers sampled the sky brightness at irregular intervals as sky conditions warranted. A typical sky brightness measurement takes $30-40 \mathrm{~s}$ to perform. This is a significant fraction of a pulsation cycle, so we treat each part of the light curve not interrupted by a sky measurement as a separate run in our analysis. The observers also took special care to obtain sky measurements near pulsation minima, to preserve as many light curve maxima as pos-

TABLE 1

JOURNAL OF OBSERVATIONS

\begin{tabular}{|c|c|c|c|c|c|}
\hline Observatory & $\begin{array}{l}\text { Telescope } \\
\text { (m) }\end{array}$ & Run Name & $\begin{array}{l}\text { Date } \\
\text { (UT) }\end{array}$ & $\begin{array}{l}\text { Run Start } \\
\text { (UT) }\end{array}$ & $\begin{array}{l}\text { Run Length } \\
\text { (s) }\end{array}$ \\
\hline Suhora $\ldots \ldots \ldots \ldots$ & 0.6 & G-0224 & 1992 Feb 25 & $1: 40: 30$ & 9695 \\
\hline Suhora $\ldots \ldots \ldots \ldots$ & 0.6 & G-0225 & 1992 Feb 25 & $20: 04: 40$ & 6275 \\
\hline Suhora..$\ldots \ldots \ldots$ & 0.6 & G-0225a & 1992 Feb 25 & $22: 26: 50$ & 20080 \\
\hline Suhora ........... & 0.6 & G-0226 & $1992 \mathrm{Feb} 26$ & $18: 03: 30$ & 5125 \\
\hline Suhora .............. & 0.6 & G-02262 & 1992 Feb 26 & $20: 03: 30$ & 28145 \\
\hline Kavalur .................. & 2.3 & K92-0162 & 1992 Feb 26 & $22: 00: 40$ & 7325 \\
\hline Suhora ........... & 0.6 & G-0227 & $1992 \mathrm{Feb} 27$ & $20: 55: 20$ & 4005 \\
\hline Kavalur ........... & 2.3 & K92-0164 & $1992 \mathrm{Feb} 27$ & $21: 32: 30$ & 8005 \\
\hline Suhora ............. & 0.6 & G-02272 & 1992 Feb 28 & $1: 14: 50$ & 10405 \\
\hline McDonald ........ & 0.9 & RA254 & $1992 \mathrm{Feb} 28$ & 7:44:00 & 17240 \\
\hline Mauna Kea...... & 0.5 & CFC-0075 & $1992 \mathrm{Feb} 28$ & $13: 19: 20$ & 8585 \\
\hline Suhora ........... & 0.6 & G-0228 & $1992 \mathrm{Feb} 28$ & $19: 00: 20$ & 32175 \\
\hline Kavalur .............. & 2.3 & K92-0166 & 1992 Feb 28 & $21: 26: 50$ & 4730 \\
\hline Kavalur ................... & 2.3 & K92-0167 & $1992 \mathrm{Feb} 28$ & $22: 58: 30$ & 3425 \\
\hline McDonald ........ & 0.9 & RA259 & $1992 \mathrm{Feb} 29$ & $7: 19: 40$ & 18580 \\
\hline Kavalur ............ & 0.9 & K42-0169 & $1992 \mathrm{Feb} 29$ & $21: 50: 20$ & 7670 \\
\hline Suhora $\ldots \ldots \ldots \ldots$ & 0.6 & G-0229 & 1992 Mar 1 & $2: 12: 30$ & 6730 \\
\hline McDonald ........ & 0.9 & RA263 & 1992 Mar 1 & $7: 09: 50$ & 5490 \\
\hline Suhora ........... & 0.6 & G-0230 & 1992 Mar 1 & $17: 54: 30$ & 36715 \\
\hline Kavalur ........... & 0.9 & K42-0171 & 1992 Mar 1 & $21: 10: 30$ & 5605 \\
\hline McDonald ........ & 0.9 & RA267 & 1992 Mar 2 & $8: 19: 30$ & 4680 \\
\hline McDonald ......... & 0.9 & RA268 & 1992 Mar 2 & $9: 37: 40$ & 6995 \\
\hline Kavalur ........... & 0.9 & K42-0174 & 1992 Mar 2 & $21: 12: 20$ & 785 \\
\hline Kavalur ........... & 0.9 & K42-0175 & 1992 Mar 2 & $21: 48: 30$ & 3665 \\
\hline Kavalur ........... & 0.9 & K42-0176 & $1992 \mathrm{Mar} 2$ & $22: 58: 10$ & 3365 \\
\hline Kavalur ............ & 0.9 & K42-0179 & 1992 Mar 3 & $20: 42: 00$ & 10820 \\
\hline McDonald ......... & 0.9 & RA270 & 1992 Mar 4 & $8: 42: 40$ & 13595 \\
\hline McDonald ........ & 0.9 & RA272 & 1992 Mar 5 & $6: 52: 30$ & 9895 \\
\hline Suhora ............. & 0.6 & G-0231 & 1992 Mar 5 & $18: 05: 00$ & 35440 \\
\hline Mauna Kea....... & 0.5 & CFC-0089 & 1992 Mar 5 & $10: 54: 10$ & 17820 \\
\hline McDonald ........ & 0.9 & RA274 & 1992 Mar 6 & $9: 00: 40$ & 11375 \\
\hline Mauna Kea....... & 0.5 & CFC-0093 & 1992 Mar 6 & $10: 52: 10$ & 17605 \\
\hline Suhora ............ & 0.6 & G-0232 & 1992 Mar 6 & $23: 28: 30$ & 16315 \\
\hline McDonald ........ & 0.9 & RA276 & $1992 \operatorname{Mar} 7$ & $8: 40: 00$ & 11735 \\
\hline Suhora $\ldots \ldots \ldots \ldots$ & 0.6 & G-0233 & 1992 Mar 7 & $18: 11: 20$ & 34655 \\
\hline McDonald ........ & 0.9 & RA280 & 1992 Mar 9 & $8: 17: 10$ & 12815 \\
\hline Suhora ............ & 0.6 & G-0234 & 1992 Mar 9 & $18: 18: 00$ & 34015 \\
\hline
\end{tabular}


sible for eventual rate of period change measurements. We use blue-sensitive bi-alkalai phototubes in all of our photometers without intervening filters to maximize the signal-to-noise ratio, as we are searching for low-amplitude pulsations. We collect our data in contiguous $5 \mathrm{~s}$ integrations.

We reduce the data in the manner described by Nather et al. (1990), and place all of our data on a common time and fractional amplitude scale. We do not, however, attempt to correct for differing signal-to-noise ratios that result from the different instrument/telescope combination used; most telescopes were between 0.6 and $1.0 \mathrm{~m}$ in diameter, so these effects are not severe. After removing the effects of sky background and extinction, we then subtract the mean light level of the star from each run, and derive light curves in terms of the fractional amplitude. This method allows us to use the star as its own magnitude calibration for each telescope. Places where the light curve from two different sites overlap show good agreement.

We use the intensity, amplitude, and power units described in Winget et al. (1994). We use millimodulation intensity (mmi) for units of our light curves and millimodulation amplitude (mma) and micromodulation power $(\mu \mathrm{mp})$ as amplitude and power units in our Fourier transform (FT) spectra.

Because our coverage is not continuous, we use the spectral window to determine the amount of spectral leakage (aliasing) created by data gaps. We create a spectral window by sampling a single, noise-free sine curve in the same way as the data and compute its power spectrum. This "forest" of peaks is what we can expect to find in the power spectrum of our data when only a single frequency is present in the light curve. We calculated a FT of the data set for the region around $109 \mathrm{~s}$ and display it in Figure 1, along with the spectral window. Our coverage was quite good during the central part of the run, as evidenced by the small size of the side lobes. The largest side lobes are due to $0.5,1$, and 2 day data gaps.

\section{ANALYSIS}

The FT of our data set shows two peaks dominating the power spectrum, along with a cluster of three nearly equal size peaks in between. The complex spectral window hides the fact that only the outer two peaks and the central peak (at $109.47 \mathrm{~s}$, $109.28 \mathrm{~s}$, and $109.09 \mathrm{~s}$ ) are real. If we subtract three sinusoids with periods, amplitudes, and phases given by the FT from the original data set, there are no peaks remaining with amplitudes greater than $0.26 \mathrm{mma}$ (see Fig. 2). We find no evidence for significant peaks other than the $109 \mathrm{~s}$ triplet in the FT. This is consistent with the findings of KRN.

The complete FT is presented in Figure 2. We check the reality of the remaining peaks below $0.26 \mathrm{mma}$ around $109 \mathrm{~s}$ and the peaks below $0.35 \mathrm{mma}$ above $350 \mathrm{~s}$ by reordering the data points at random (shuffling the data set). This removes any time series correlation present; by taking the FT of many shuffled data sets, we determine the likelihood that a given peak in our original FT arises by chance. For our shuffled data sets, we find no peaks above $10\langle P\rangle$, where $\langle P\rangle$ is the local average power in the FT (Horne \& Baliunas 1986). This result agrees with the "false alarm probability" of Scargle (1982), using the number of independent frequencies (number of frequencies separated by $\Delta f$, Press \& Rybicki 1989) calculated from the true sampling of the data set, i.e., $\Delta f=(\Delta T)^{-1}=0.77$ $\mu \mathrm{Hz}$, where $\Delta T$ is the total time span of the data set. In Figure 3 , we present the histogram of peaks above $n \times\langle P\rangle$, for the real and shuffled data sets for different values of $n$. The average

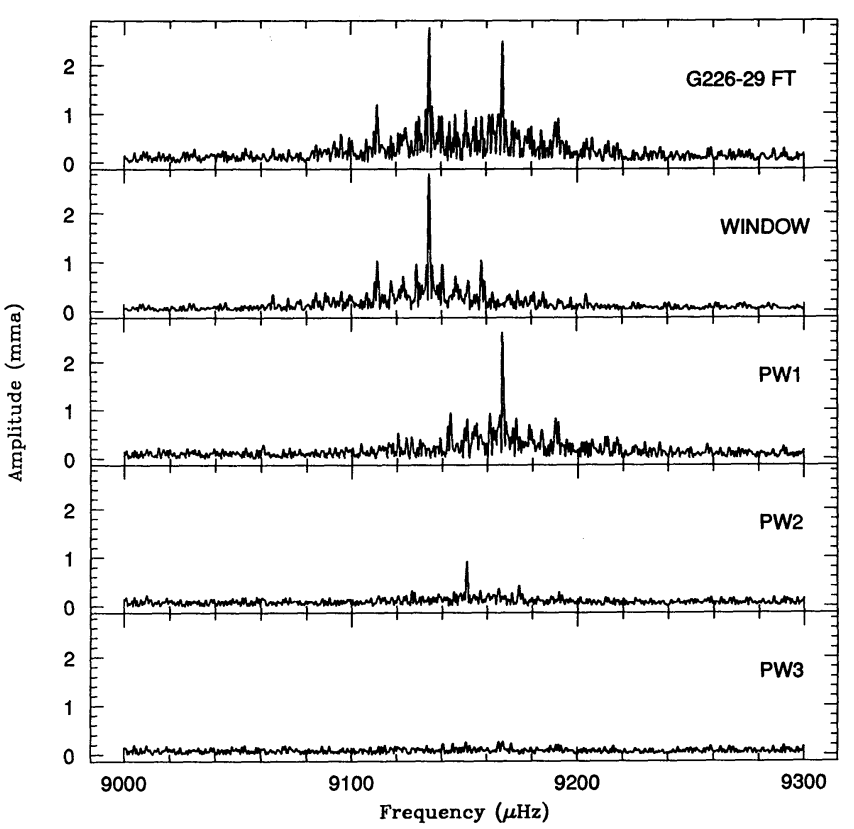

Fig. 1.-The amplitude spectra of the total light curve of G226-29, for the region of the $109 \mathrm{~s}$ triplet (peak above the dashed line in Fig. 2). The upper panel is the Fourier spectra of the data. The second panel (WINDOW) is the spectra window, i.e., the transform of a noiseless sine curve. PW1 is the Fourier spectra after removing (prewhitening) one sine-curve with $P=109.4724 \mathrm{~s}$, and amplitude of $2.8 \mathrm{mma}$. PW2 is the Fourier spectra after removing (prewhitening) a second sine curve with $P=109.0867 \mathrm{~s}$, and amplitude of 2.5 mma. PW3 is the Fourier spectra after removing (prewhitening) a third sine curve with $P=109.2784 \mathrm{~s}$, and amplitude of $1.1 \mathrm{mma}$.

power around $350 \mathrm{~s}$ is $0.012 \mu \mathrm{mp}$ and around $109 \mathrm{~s}$ is 0.008 $\mu \mathrm{mp}$. By definition of the false alarm probability, the average power includes the real peaks, but the average is taken in a large region to preclude the domination of the average power by the real peaks and their aliases.

\section{RESULTS AND DISCUSSION}

Our analysis shows the triplet of peaks near $109 \mathrm{~s}$ are the only significant pulsation modes in G226-29. A simultaneous nonlinear least-squares fit of three sinusoids to our data set yields the following periods, amplitudes, and time of maxima:

$$
\begin{gathered}
P_{+1}=109.08672 \pm 0.00022 \mathrm{~s}, \quad A_{+1}=2.49 \pm 0.10 \mathrm{mma}, \\
\text { BJDD }_{\max }^{+1}=2448678.789954 \pm 1.3 \mathrm{~s} \\
P_{0}=109.27838 \pm 0.00051 \mathrm{~s}, \quad A_{0}=1.08 \pm 0.10 \mathrm{mma} \\
\text { BJDD }_{\max }^{0}=2448678.789361 \pm 3.0 \mathrm{~s}, \\
P_{-1}=109.47239 \pm 0.00019 \mathrm{~s}, \quad A_{-1}=2.82 \pm 0.10 \mathrm{mma}, \\
\text { BJDD }_{\max }^{-1}=2448678.789540 \pm 1.1 \mathrm{~s} .
\end{gathered}
$$

All the periods are within $1 \sigma$ of the values given by KRN. The amplitudes of the $P_{-1}$ and $P_{0}$ modes are within $2 \sigma$ of the values given by KRN, but the amplitude of the $P_{+1}$ mode is smaller by $5 \sigma$ now. The amplitudes are our least precise determinations, because we do not have three channel data that would allow us to more accurately subtract out sky brightness variations. Our experience suggests the amplitude uncertainty is about $10 \%$. Based on this, we do not consider the amplitude changes with time of the $P_{0}$ or $P_{-1}$ modes to be significant, but it is marginally significant for the $P_{+1}$ mode. 


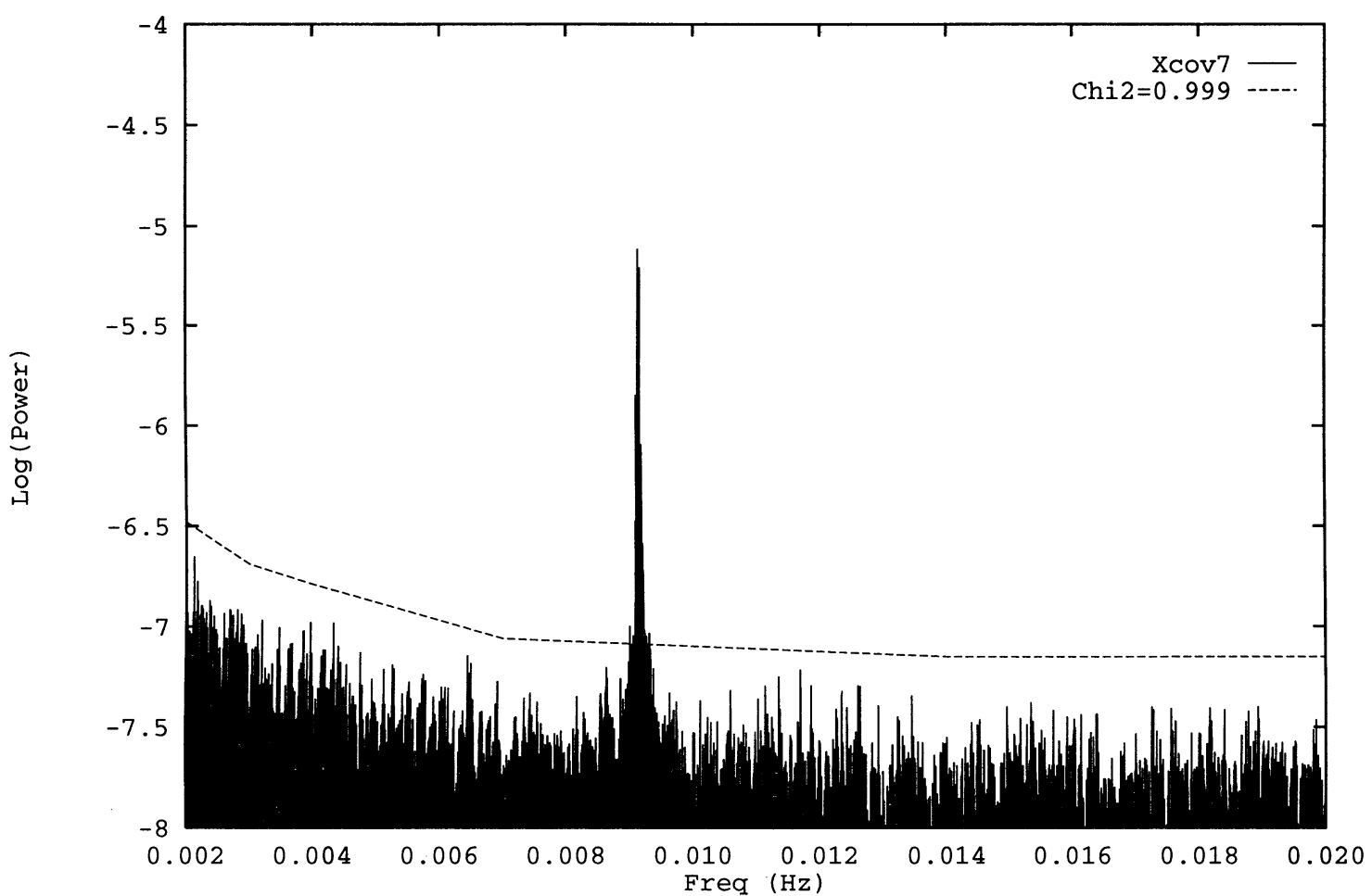

FIG. 2.-The amplitude spectrum (Fourier transform) of the total light curve of G226-29. The dashed line represents the false alarm probability of $1 / 1000$, i.e., any peak above the line has a probability less than one chance in 1000 of being due to noise.

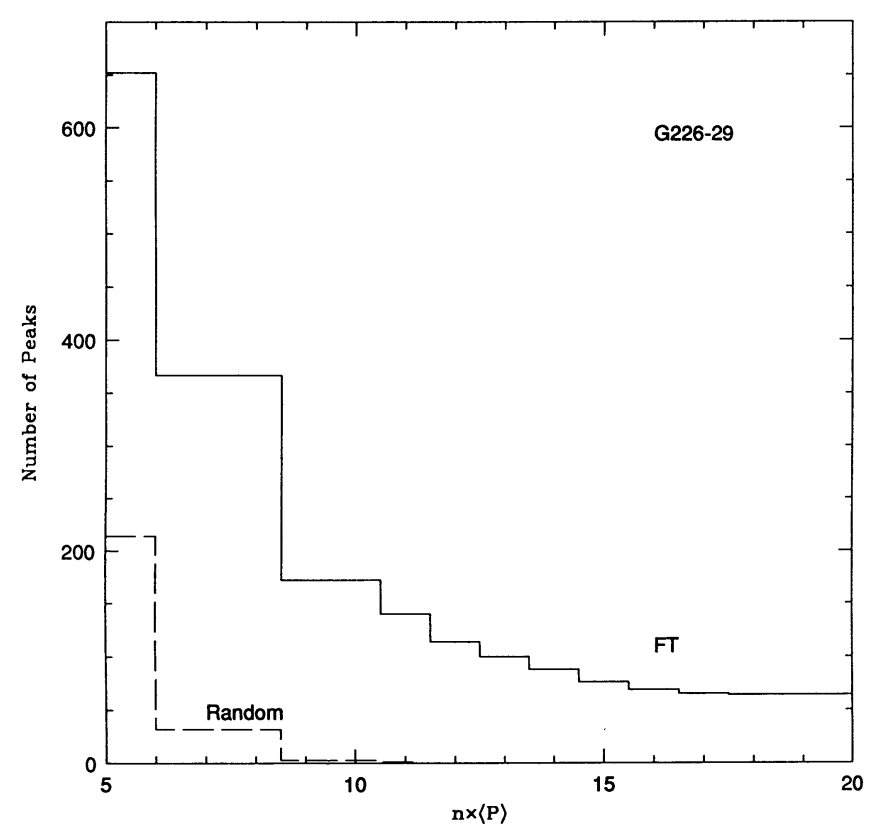

FIG. 3.-Histogram of the number of peaks in the Fourier transform of the light curve of G226-29 above $n \times\langle P\rangle$. All peaks above $10\langle P\rangle$ are in the region of the $9135 \mu \mathrm{Hz}$ peak (109 s). The number of peaks above $10\langle P\rangle$ is larger than 3 because of the aliases (spectral window). The dashed line is the histogram for the FT of the shuffled data set. No peaks above $10\langle P\rangle$ are seen on the shuffled data set.
The frequency splittings between the members of the triplet are $16.1 \mu \mathrm{Hz}$ for $\left(P_{+1}-P_{0}\right)$ and $16.2 \mu \mathrm{Hz}$ for $\left(P_{0}-P_{-1}\right)$. The time span of the run was too short to consider this frequency difference significant. Even if it were significant, it goes in the wrong direction to be explained by the presence of a magnetic field (see Winget et al. 1994, for example). Our null result does not agree with that of Angel, Borra, \& Landstreet (1981), who reported a magnetic field of $B=(1.7 \pm 0.8) \times 10^{5} \mathrm{G}$, but Jones et al. (1989) state this $2 \sigma$ detection could easily be a null result with a slightly different interpretation of the errors.

The most obvious explanation for an evenly split $g$-mode triplet is that of a single $l, k$ nonradial pulsation mode split into the three possible components by slow rotation. We consider the $l=1$ mode identification as the most probable for three reasons. First, we have secure $l$ identifications for the DBV star GD 358 and the DOV star PG 1159-035, and find that the $l=1$ modes (triplets) dominate the power. Second, Robinson et al. (1995) demonstrated that $l=1$ for G117-B15A, the only DAV to have a secure mode identification-G117-B15A is also located at the blue edge of the instability strip. Finally, Clemens (1994) presents convincing evidence that essentially all power observed in the best-studied DAVs is the result of $l=1$ pulsations, suggesting that an a priori assumption of $l=1$ pulsations is plausible. Assuming we have an $l=1, k=1$ mode, we can then relate the observed splitting to the rotation period of the white dwarf with the relation (Unno et al. 1989)

$$
\delta \sigma_{l k m}=\delta m \Omega_{\text {rot }}\left(1-C_{l k}\right),
$$

where $C_{l k}$ is the first-order splitting coefficient and is most dependent on $l$. We use $C_{l k}$ values for modes near $109 \mathrm{~s}$ from models of Bradley (1995); even though the asymptotic value is valid only for large $k$, the values from the models are close to the asymptotic value of $[l(l+1)]^{-1}$ for $C_{l k}$. Using an average 
splitting of $16.15 \mu \mathrm{Hz}$ and the appropriate $C_{l k}$ value of 0.48 $(l=1, k=1)$, we derive a rotation period of $8.9 \mathrm{hr}$.

The differing amplitudes of the modes within the $109 \mathrm{~s}$ triplet could be the result of either differences in the amount of pulsation driving for each mode, the inclination of the pulsation axis to our line of sight, or both. Here, we assume the pulsation driving differences are small, and use the relative amplitudes of the pulsation modes to derive the inclination of the pulsation axis. The ratio of the central peak to the two outlying peaks lies between 0.43 and 0.38 . Using the results of Pesnell (1985) for $l=1$, we find the pulsation axis is tilted between $70^{\circ}-75^{\circ}$ to our line of sight, i.e., nearly equator-on. This result is consistent with what we derive using the amplitudes of KRN. The similarity of the amplitudes from 1980 to 1982 and 1992 and the similarity of the amplitudes of the $P_{-1}$ and $P_{+1}$ modes suggests that most of the amplitude differences within the triplet are due to the inclination of the pulsation axis.

The richness of the theoretical $g$-mode spectrum demands we make assumptions about the effective temperature and mass of G226-29 if we wish to constrain the hydrogen layer mass. In our analysis, we use $\sim 0.70 M_{\odot}(\log g \approx 8.2)$ models, with effective temperatures near either $13,600 \mathrm{~K}$ or $12,100 \mathrm{~K}$. Fontaine et al. (1992) compared the triplet found by KRN to the grid of adiabatic pulsation periods computed by Brassard et al. (1992) for white dwarf models. They show the star must have a thick hydrogen layer $\left(\log q=\log M_{\mathrm{H}} / M_{*}=-4.4\right.$ $\pm 0.2)$ if the observed triplet is the $l=1, k=1$ mode. Bradley $(1993,1995)$ obtains similar results using theoretical periods from a different grid of pure carbon core evolutionary white dwarf models. More recently, Bradley \& Clemens (1995) computed a grid of evolutionary white dwarf models with oxygenrich cores and $M_{\mathrm{H}}=1.5 \times 10^{-4} M_{*}(\log q=-3.8)$ to explain the pulsation spectra of the hotter ZZ Ceti stars with resolved pulsation spectra. With these models, a $0.75 M_{\odot}$ model matches the $109 \mathrm{~s}$ period at $13,300 \mathrm{~K}$, and they require a 0.78 $M_{\odot}$ model for a match at $12,300 \mathrm{~K}$. If we use the parallax as an additional constraint, the $0.75 M_{\odot}$ models of Bradley \& Clemens (1995) near $13,300 \mathrm{~K}$ have luminosities consistent with the parallax; the $0.78 M_{\odot}$ model has a parallax that is $2 \sigma$ too low.

The two most likely spectroscopic effective temperature determinations do not place G226-29 near the theoretical blue edge of Bradley \& Winget (1994) for $0.70 M_{\odot}$ models, which is $\sim 12,900 \mathrm{~K}$. The blue edges of Bradley \& Winget are for models with very efficient convection; the location of the blue edge can easily be $1000 \mathrm{~K}$ cooler with less efficient convection. We believe we are seeing an inefficiently driven $l=1$, $k=1$ pulsation mode, because linear growth rates for this mode are small (Bradley 1993). Here any $l=2$ or 3 modes would not be excited to observable amplitudes by a combination of inefficient pulsation driving and geometric cancellation effects. This interpretation suggests the effective temperature is near $13,000 \mathrm{~K}$, assuming efficient convection. We cannot rule out a cooler temperature for G226-29 and for the blue edge; we would require a less efficient version of convection if this is the case. This may well be the case, because Koester, Allard, \& Vauclair (1994) shows G226-29 must have nearly the same temperature of L19-2 and G117-B15A, at about $12,400 \mathrm{~K}$, in agreement with Kepler \& Nelan (1993) and
Koester \& Allard (1993). Fortunately, there are observational tests to confirm the $l=1$ identity of the $109 \mathrm{~s}$ triplet. Fontaine et al. (1992) suggest using two-color photometry observations during a pulsation cycle, because different $l$ values have different patterns. Robinson et al. (1995) used the differences in the time-resolved $H S T$ ultravoiolet photometry to derive the pulsation index $l$ for G117-B15A.

Were it available, we could use the rate of period change to determine the combination of the stellar mass and core composition. The models of Bradley $(1993,1995)$ suggest that $d P / d t$ for the $109 \mathrm{~s}$ modes due to secular evolution is about $1 \times 10^{-15} \mathrm{~s} \mathrm{~s}^{-1}$. For this $d P / d t$, a detection will require about $20 \mathrm{yr}$ of phase-resolved data. Although a dauntingly long-term project, the simplicity of the pulsation spectrum makes it much easier to detect $d P / d t$ here than in a complex pulsator. Continued monitoring of this star is clearly worthwhile.

\section{SUMMARY AND CONCLUSIONS}

First, we summarize the pulsation properties of G226-29 revealed by our observations. We see no evidence of other periodicities other than the known $109 \mathrm{~s}$ triplet, down to a limit of about $0.3 \mathrm{mma}$. The periods of the $109 \mathrm{~s}$ triplet members are within $1 \sigma$ of the values of KRN; the amplitudes of the $P_{0}$ and $P_{-1}$ modes are within $2 \sigma$ of KRN's values, while the $P_{+1}$ mode has an amplitude about $5 \sigma$ lower.

Different reported spectroscopic observations suggest the mass of $\mathrm{G} 226-29$ is near $0.70 M_{\odot}$, but disagree on the temperature. It is either near $13,600 \mathrm{~K}$ or near $12,100 \mathrm{~K}$. We must constrain the $l$ and $k$ value of the observed mode to determine the likely structure of G226-29. If we assume we are seeing an $l=1, k=1$ mode, then the hydrogen layer mass is about $10^{-4}$ $M_{*}$, in general agreement with the results of Fontaine et al. (1992), and the effective temperature is near $13,000 \mathrm{~K}$, near the blue edge of the instability strip for models with efficient convection.

If we assume the unequal amplitudes of the triplet are due solely to the inclination of the pulsation axis to our line of sight, we find the inclination is between 70 and $75^{\circ}$ for an $l=1$ mode. Theoretical rates of period change for modes near $109 \mathrm{~s}$ are near $1 \times 10^{-15} \mathrm{~s} \mathrm{~s}^{-1}$, implying that we need another $20 \mathrm{yr}$ of phase-resolved data to detect it. The uncomplicated pulsation spectrum makes this a relatively simple, though time consuming, task.

Once observations that confirm the $l=1$ identity of the 109 $s$ triplet and the effective temperature are in hand, we will have good constraints on the stellar mass, hydrogen layer mass, rotation rate, and possibly the convective efficiency of G226-29. In spite of the simple pulsation spectrum, we can constrain the structure of G226-29 surprisingly well.

This work was supported by the National Science Foundation under grant AST 90-14655 through the University of Texas and McDonald Observatory, AST 92-17988, through the Florida Institute of Technology, from grants from FINEP and CNPq in Brazil, and grant no. 2-2109-91-02 from KBN in Poland. The calculations were performed with the Cray supercomputer of the Centro Nacional de SupercomputaçãoUFRGS, Brazil. 


\section{REFERENCES}

Angel, J. R. P., Borra, E. F., \& Landstreet, J. D. 1981, ApJS, 45, 457

Bergeron, P., Wesemael, F., \& Fontaine, G. 1992, ApJ, 387, 288

Bradley, P. A. 1993, Ph.D. thesis, Univ. Texas

.1995, ApJ, submitted

IHI Bradley, P. A., \& Clemens, J. C. 1995, ApJ, submitted

Bradley, P. A., \& Winget, D. E. 1994, ApJ, 421, 236

Bradley, P. A., Winget, D. E., \& Wood, M. A. 1989, in IAU Colloq. 114, White Dwarfs, ed. G. Wegner (Berlin: Springer), 286

Brassard, P., Fontaine, G., Wesemael, F., \& Tassoul, M. 1992, ApJS, 81, 747

Clemens, J. C. 1994, Ph.D. thesis, Univ. Texas

Cox, A. N., Starrfield, S. G., Kidman, R. B., \& Pesnell, W. D. 1987, ApJ, 317, 303 Daou, D., Wesemael, F., Bergeron, P., Fontaine, G., \& Holberg, J. B. 1990, 364,

Fontaine, G., Brassard, P., Bergeron, P., \& Wesemael, F. 1992, ApJ, 399, L91

Fontaine, G., Brassard, P., \& Wesemael, F. 1994, ApJ, 428, L61

Harrington, R. S., \& Dahn, C. C. 1980, AJ, 85, 454

Horne, J. H., \& Baliunas, S. L. 1986, ApJ, 302, 757

Jones, P. W., Pesnell, W. D., Hansen, C. J., \& Kawaler, S. D. 1989, ApJ, 336, 403

Kepler, S. O., \& Nelan, E. P. 1993, AJ, 105, 608
Kepler, S. O., Robinson, E. L., \& Nather, R. E. 1983, ApJ, 271, 744 (KRN)

Koester, D., \& Allard, N. 1993, in White Dwarfs: Advances in Observations and Theory, ed. M. A. Barstow (Dordrecht: Kluwer), 237

Koester, D., Allard, N. F., \& Vauclair, G. 1994, AA, 291, L9

McGraw, J. T., \& Fontaine, G. 1980, unpublished results

Nather, R. E. 1973, Vistas Astron., 15, 91

Nather, R. E., Winget, D. E., Clemens, J. C., Hansen, C. J., \& Hine, B. P. 1990 ApJ, 361, 309

Pesnell, W. D. 1985, ApJ, 292, 238

Press, W. H., \& Rybicki, G. B. 1989, ApJ, 338, 277

Robinson, E. L., Kepler, S. O., \& Nather, R. E. 1982, ApJ, 259, 219

Robinson, E. L., Mailloux, T. M., Zhang, E., Koester, D., Stiening, R. F., Bless, R. C., Percival, J. W., Taylor, M. J., Dolan, J. F., \& van Citters, G. W. 1995 ApJ, 438, 908

Scargle, J. D. 1982, ApJ, 263, 835

Unno, W., Osaki, Y., Ando, H., Saio, H., \& Shibahashi, H. 1989, Nonradial Oscillations of Stars (2d ed.; Tokyo: Univ. Tokyo Press)

Winget, D. E., et al. 1994, ApJ, 430, 839

Winget, D. E., Van Horn, H. M., Tassoul, M., Hansen, C. J., Fontaine, G., \& Carroll, B. W. 1982, ApJ, 252, L65 